\title{
Untersuchungen zur Morphogenese im Katmandu Valley
}

Mit Unterstützung des Schweizerischen Nationalfonds zur Förderung der wissenschaftlichen Forschung konnten frühere Untersuchungen zur Morphologie von Nepal in den Jahren 1971 bis 1973 fortgeführt werden ${ }^{1)}$. Sie dienten vor allem dazu, die Morphogenese des Tales von Katmandu, das einst von einem See erfüllt war, zu klären. Die geologische Geschichte fand ihren Niederschlag auch in der Mythologie ${ }^{2)}$ und bestimmte bis heute gewisse Verhaltensweisen der ansässigen Bevölkerung.

Im englischen Sprachgebrauch nennt man das etwa $30 \times 20 \mathrm{~km}$ weite Talbecken meistens das Valley. In die Untersuchungen wurde das ganze Valley einbezogen; die morphologische Detailkartierung konzentrierte sich jedoch auf den für die morphologische Deutung wichtigen südlichen Teil, wo der heilige Bagmati Fluß das Valley verläßt und durch wilde Schluchten die Mahabharat Ketten querend der indischen Tiefebene zustrebt.

Nachdem über verschiedene Einzelfragen in den oben erwähnten Arbeiten regelmäßig Berichte über die Ergebnisse der Feldarbeiten erschienen, faßt die vorliegende Arbeit auf der einen Seite den heutigen Stand in der Weise zusammen, daß die wichtigsten Fragen Schritt für Schritt anhand von Figuren besprochen und auf der anderen Seite eine Reihe von noch offenen Problemen im Hinblicke auf zukünftige Forschungen erwähnt werden.

Figur 1 bringt die generalisierte Wiedergabe eines großen Teiles der morphologischen Feldkartierung $1: 63630$. Am oberen Kartenrand liegt Katmandu, das hydrographische Zentrum des Valley. Der Bagmati entspringt in der Nordost-Ecke des Valley. Er nimmt in der Gegend von Katmandu die meisten zentripetal hier zusammenfließenden Zuflüsse auf. Wenig unterhalb der Hauptstadt durchbricht er die Felsrippe von Kirtipur-Chobhar in einer ganz im Anstehenden eingeschnittenen Schlucht. Dieser Punkt spielt für die Entwässerung des Valley die Rolle der lokalen Erosionsbasis. Unterhalb beginnt sich der Bagmati rasch in die weichen Seeablagerungen einzuschneiden. Bei Katmandu liegt der Bagmati bei $1276 \mathrm{~m}$ ü. M. Der Punkt, an welchem er das Valley verläßt, liegt noch etwa $1220 \mathrm{~m}$ ü. M. Die Legende der Kartenskizze ist bei der Figur angegeben und wird von Fall zu Fall im Laufe der Darlegungen noch eingehender erläutert werden.
Während die geologischen Verhältnisse seit langem Aufmerksamkeit fanden, gab es, als diese Untersuchungen begonnen wurden, nur wenige Arbeiten zur Morphologie; sie waren zudem oft von beschränktem wissenschaftlichen Wert, weil die Angaben nicht überprüft werden konnten ${ }^{3)}$. In den letzten Jahren wurden im Valley wichtige Resultate bei Wasserbohrungen erzielt. $\mathrm{C}^{14}$-Datierungen und pollenanalytische Bestimmungen liegen vor. Verschiedene Organisationen verfaßten Berichte, in welchen morphologisch wichtige Angaben enthalten sind. Diese Berichte sind in der Regel nicht publiziert worden und ihr Auffinden in irgend einem Amt in Katmandu ist oft langwieriger als die morphologische Feldarbeit ${ }^{4}$.

Die Übersichtskarte Figur 2 zeigt vorerst die geologisch-morphologische Zonierung nach $\mathrm{HAGEN}^{5}$ ). (b) ist die junggefaltete Zone der Randketten der Siwaliks und Mahabharat Lekh, die bis $3000 \mathrm{~m}$ Höhe unvermittelt aus der Gangesebene aufsteigen; jungpleistozäne Bewegungen können mit den üblichen geologischen Beweisverfahren an ihrem Südrande nachgewiesen werden. (c) ist das nepalische Mittelland, eine Bezeichnung, der relative Bedeutung (Mahabharat Ketten im Süden, über $8000 \mathrm{~m}$ hohe Himalaya-Hauptkette im Norden) zukommt. Das Mittelland weist enorme Höhenunterschiede auf: Die

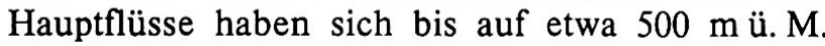
eingeschnitten, dazwischen liegen Gebirge von 3000 und mehr Meter Höhe. (d) ist die Hauptkette des Himalaya und die nördlich folgenden Hochflächen und Gebirge in Tibet.

Die Grenze (b/c) erscheint auf den geologischen Karten neuesten Datums ${ }^{6}$ ) nicht, während sie bei HAGEN $^{7)}$ besonders deutlich eingezeichnet ist. Der Gegensatz ist nur scheinbar und rührt von der unterschiedlichen Fragestellung her. Wir werden in der Folge von dieser Grenzfläche als der Scherfläche von Dakshinkhali sprechen. Sie ist eine Scherfläche, an der die südlichen Teile bei jungorogenen Bewegungen kräftig und mit einer leichten Tendenz nordwärts emporgeschoben wurden. Dakshinkhali ist ein heiliger Ort unweit von Pharping, wo der Charakter dieser Scherfläche besonders gut studiert werden

Prof. Dr. H. Boesch, Geogr. Inst. Universität Zürich, Blümlisalpstraße 10, 8006 Zürich 
Legende zu Fig.1

${ }_{02}$ " Koordination der Karte 1:63630

-ị- $\quad$ Ausgewählte Tiefbohrungen, welche das Anstehende nicht erreichten:

No. 1 Harasidhi $-457,20 \mathrm{~m}$

No. 16 Lagan Tole - $476 \mathrm{~m}$ (Ground Water Invest.Project, HMG 1970)

Heutige Entwässerung

Kalimati

* Terrassen der beiden Systeme der zweiten Erosionsphase

$\rightarrow$ Patanschotter; dazugehörige heute tote Entwässerungsrinnen

Ältere Schotter und Gehängeschutt mit Roterdebeimischung, Serie von Pharping; dazugehörige heute tote Entwässerungsrinnen

iis: Schwemmkegel, der See-AkumulationsiiN fläche aufliegend Überläufe über die Schwelle KirtipurChobhar zur Zeit der Aufstauung des Sees

Valleysedimente: Mergel

Valleysedimente: Glimmersande

Valleysedimente: grobblockige Randfazies

Roterdeniveau (in situ)

\section{Eckfluren}

Anstehendes Gestein versch. Alters (undiff.) 
Fig. 1: Morphologische Übersichtskarte des sw Katmandu Valley.

Fig. 1

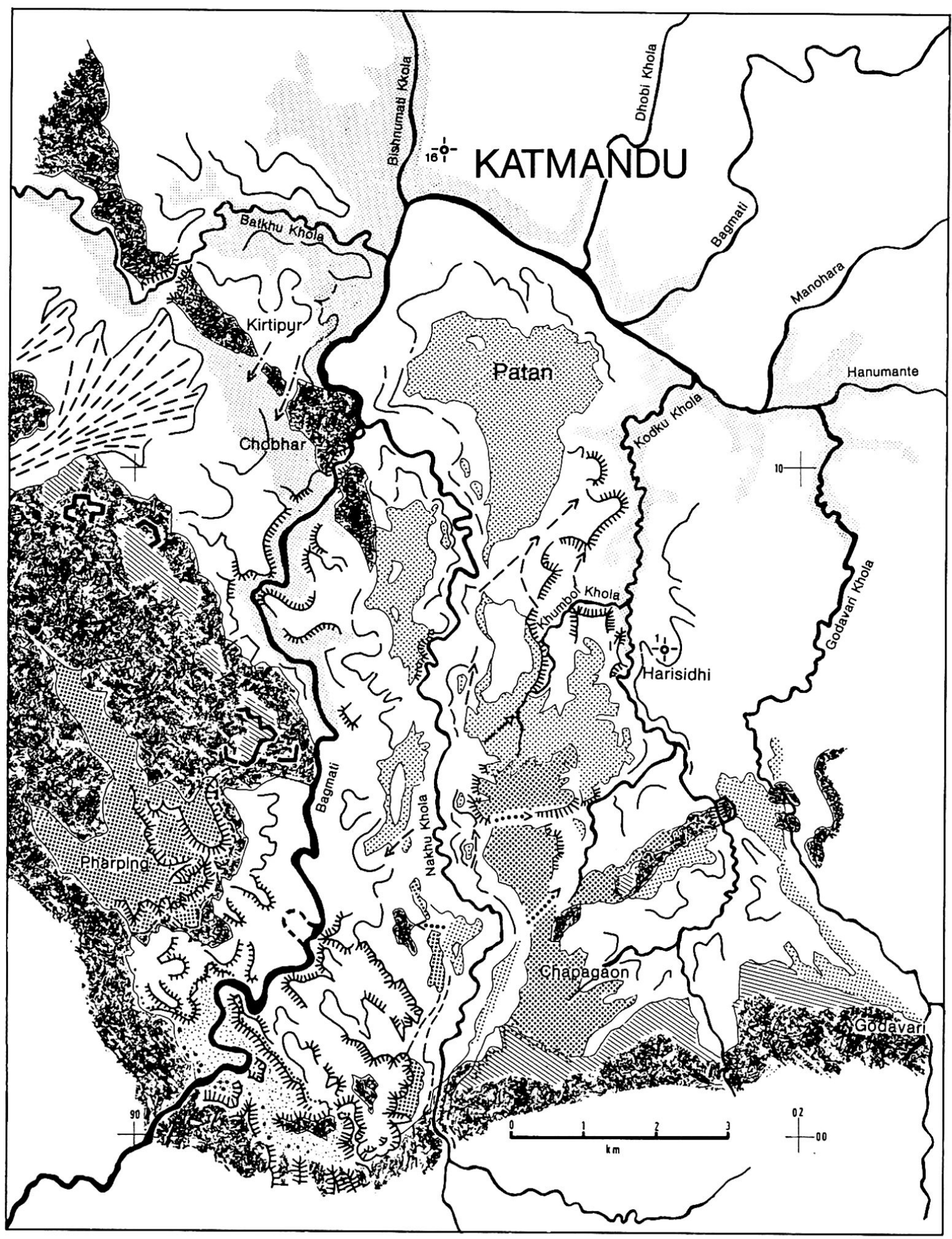


kann. Sie stellt im Vergleich mit den eigentlichen geologischen Gebirgsstrukturen (Decken usw.) eine geringfügige Störung dar und in geologischen Arbeiten darf sie durchaus vernachlässigt werden. Auf die morphologische Entwicklung hat sie sich dagegen sehr stark ausgewirkt und gewinnt damit gegenüber den geologischen Strukturen die Priorität ${ }^{8)}$.

Die Scherfläche von Dakshinkhali trennt nicht zwei verschiedene geologische Komplexe, wie alle geologischen Darstellungen des Gebietes von Katmandu zeigen. Im übrigen sind diese sehr widersprüchlich. Bei GANSSER erscheint das Süd-Nordprofil durch das Valley als Antiklinale, bei HAGEN eher als Synklinale ${ }^{9)}$. Unseren Beobachtungen und Auffassungen entspricht am ehesten ein Profil, wie es in einem vorläufigen Bericht zur Wasserversorgung von Katmandu gezeichnet wurde ${ }^{10}$.

Im übrigen sind die Details der geologischen Strukturen in unserem Zusammenhang weniger wichtig als diese nur noch in geringem Maße verändernden spätorogenen Bewegungen. Diese Bewegungen können in der Stratigraphie der Valleysedimente und der Morphogenese erfaßt und zeitlich eingestuft werden. Wenn wir in der Folge vom Anstehenden sprechen, meinen wir das Gebirge unter Ausschluß der Valleysedimente. Wir berücksichtigen seine stratigraphisch-petrographische Gliederung nur soweit es in unserem Zusammenhange sinnvoll ist.

Die jungen Bewegungen haben nicht nur die Verhältnisse im Valley, sondern ganz allgemein die Talbildung im Himalaya beeinflußt. HAGEN, der stark von R. STAUB beeinflußt war ${ }^{11}$, hat diesen Problemen besondere Aufmerksamkeit geschenkt. In der erwähnten Arbeit gibt er eine gute Übersicht über frühere Deutungsversuche zusammen mit eigenen $\mathrm{Hy}$ pothesen. Wir sind mit ihm der Auffassung, daß die die Zonen (c) und (d) mehr oder weniger parallel querenden Himalayaflüsse mit Bezug auf die Hauptfaltungsphase antezedent sind. Zur Frage, wieweit vororogene Strukturen ihren Lauf vorzeichneten, möchten wir uns hier nicht äußern. An der Grenzfläche $(b / c)$ wurden sie durch die junge Heraushebung der Zone (b) aufgehalten und nach Osten, respektive Westen abgelenkt. So wurden die beiden großen hydrographischen Einzugsgebiete (I, Narayani; II, Sun Kosi) geschaffen. Die kräftigen und raschen orogenen Bewegungen in der Zone (b) löschten bestehende ältere Talanlagen aus und schu- fen die Voraussetzung zur Bildung einer großen Zahl kleinerer, mit Bezug auf diese Faltungsphase konsequenter Flußgebiete (IV, nicht weiter unterteilt), die sich heute schon durch subsequente erweitert haben. Dies ist vor allem bei jenem Einzugsgebiet der Fall gewesen, welches sich zwischen (I) und (II) rückwärtsgreifend weiter als alle anderen in das Innere der Gebirge auszuweiten vermochte, das heißt beim Bagmati (III). Die Flußgebiete (III) und (IV) sind nicht nur kleiner, sondern vor allem auch jünger. Ältere Eintiefungssysteme sind auf (I) und (II) beschränkt. Hier sind auch die Flüsse tiefer eingeschnitten. Die Oberläufe des Bagmati im Valley sammeln sich zwischen 1250-1300 m ü. M. In gleicher Entfernung vom Gebirgsrand erreicht man jedoch westlich und östlich vom Valley über flache $\mathrm{Paß}$ übergänge die bis etwa $500 \mathrm{~m}$ ü. M. eingeschnittenen Zuflüsse von (I) und (II).

Die eben erwähnten Übergänge bilden ein Problem für sich. Sie liegen alle ungefähr auf derselben Meereshöhe ${ }^{12)}$; und diese Regelmäßigkeit allein genügt, in ihr etwas morphogenetisch Bedeutsames zu vermaten. Alle bisher aufgestellten Arbeitshypothesen scheinen aber so unsicher zu sein, daß darauf verzichtet wird, sie auch nur zu erwähnen. Sicher ist, daß im Bereich der erwähnten sieben Ausgänge keine Spuren eines allfälligen Überfließens von Wasser festgestellt werden konnte. Möglicherweise ist diese Regelmäßigkeit rein zufällig, womit sich die Suche nach einer verbindenden Ursache erübrigen würde.

Die Lösung des Problems wird noch kompliziert, weil die Ausgänge gegen Osten (Sanga Paß) vorerst in das weite Talbecken von Banepa auf etwa $1520 \mathrm{~m}$ ü. M. - also wesentlich höher als das Valley - führen. Dieses vom Punyamati Khola durchflossene Gebiet ist zwar dem Sun Kosi tributär, doch hat dieser in rückgreifender Erosion mit dem Rosi Khola erst den südlichsten Teil bei etwa $1370 \mathrm{~m}$ ü. M. erreicht. Das Becken von Banepa ist ein Teil jener alten - später zu besprechenden -, von Roterde bedeckten Oberfläche; die jungen Bewegungen haben auch hier wie im Valley nördlich der Scherfläche später einen seichten See geschaffen. Er ist noch wenig untersucht worden, dürfte aber sicher wertvolle Vergleichsresultate liefern.

HAGEN stellt - wobei er freilich auf der fraglichen Karte ein Fragezeichen anbringt - die Entwicklung 
im Falle des Bagmati (III) etwas anders dar ${ }^{13)}$. Auch hier greift er zeitlich in der Deutung weit zurück und drückt die Vermutung aus, daß (III) ebenfalls auf eine südwärts gerichtete Hauptentwässerung mit Ursprung in Zone (d) zurückgehe, also im Prinzip antezedent sei. Zweifellos sind alte Talsysteme in einer Höhe angelegt worden, die weit über der heutigen realen und beobachtbaren Oberfläche liegt. Diese Topographie mit ihren geologischen Verhältnissen kann immer nur indirekt entschlüsselt werden und alle Aussagen bleiben hypothetisch. Wenn wir hier HAGEN nicht folgen können, dann geschieht dies deshalb, weil kein einziges Indiz für diese Lösung spricht (beispielsweise korrelate Sedimente in Zone (b)). Im Zusammenhang mit seiner These erwähnt HAGEN die Wahrscheinlichkeit einer Seenbildung am Gebirgsrand und verwendet per analogiam den Begriff "Alpenrandsee». Leider ist dieser Begriff von indischen und nepalischen Geologen und Morphologen aufgegriffen und als Fremdwort im Englischen verwendet worden. Wir sagen leider, weil sie diesen Begriff unrichtig auffaßten und beispielsweise eine direkte Verbindung mit dem viel jüngeren See im Valley herstellten. Späte orogene Bewegungen können leicht zu intramontaner Seebildung führen. Meist werden diese jedoch bald durch die verstärkte Erosion wieder entleert. Nur selten sind ihre Spuren so klar zu erkennen wie bei Katmandu und Banepa. Ein ausgezeichnetes Vergleichsobjekt stellt der gut untersuchte See von Srinagar (Kaschmir) dar.

(e) bezeichnet die Staatsgrenze von Nepal und (f) die Grenzen der erwähnten hydrographischen Gebiete I-IV.

Figur 3 ist ein allgemeines Nord-Südprofil durch das Valley. Das Anstehende wird hier nur soweit gegliedert, als es für das Verständnis des Folgenden notwendig ist ${ }^{14}$. Granite und Migmatite (1) bauen hauptsächlich die im Norden gelegene Kette Sheopuri Lekh auf. Auf der nach Süden exponierten Flanke verwittern diese Gesteine besonders kräftig zu einem Grus von Quarzkörnern, Feldspäten und silberglänzenden Glimmerplättchen. Eigentliche Gerölle sind ausgesprochen selten. Dieses für die Nordseite des Valley charakteristische Verwitterungsmaterial wird in das Valley verschwemmt und sedimentiert. In allen anderen Teilen der Umgebung des Valley ist das Anstehende in stratigraphischer und petrographischer Hinsicht von großer Vielfalt (2), worüber die erwähnte geologische Karte und verschiedene Publikationen Auskunft geben. An den steilen Berghängen finden sich grobblockige Schutthalden und Schuttkegel. Die Bergbäche und Flüsse bringen wohlgerundete Schotter ins Valley, wobei die Ausdehnung der Schotterfluren und deren Mächtigkeit stark schwankt. Die für den Norden typische Glimmerführung fehlt vollkommen.

Der letztgenannte Unterschied würde noch aus anderen Gründen eine sorgfältige, vermutlich interdisziplinäre Untersuchung rechtfertigen. Besucher des Valley leiden meist schon in der ersten oder zweiten Woche an Magen- und Darmstörungen, die man fatalistisch als «Katmandu Disease» bezeichnet und für die man die feinen Glimmerblättchen im $\mathrm{Ge}$ brauchswasser verantwortlich macht. Ein Autor zitiert den andern, und hartnäckig wird die Meinung vertreten, daß man diese Situation eben hinnehmen müsse - dabei kann man die Katmandu-Krankheit auch mit viel näher liegenden und vor allem mit Ursachen, die sich beheben lassen, erklären ${ }^{15}$.

Die Valleysedimente sind im ganzen nördlichen Teil, etwa bis zum Bagmati, von dieser charakteristischen Glimmersandfazies bestimmt (3). Bohrungen und Feldbeobachtungen in Taleinschnitten zeigen gegen Norden zunehmend einzelne Gerölleinlagerungen und gegen Süden ein immer stärkeres Auftreten der mergeligen Fazies (4). Diese hell- bis dunkelgrauen Mergel bilden südlich von Katmandu die Valleysedimente; vor allem südlich der Kirtipur-ChobharSchwelle sind sie in großer Mächtigkeit und eintönig ausgebildet. Erst im südlichsten Teil des Valley, wo der Bagmati die Dakshinkhali Scherfläche erreicht und sich in die große Schlucht zwängt, begegnen wir plötzlich wieder den gleichen Glimmersanden, wie wir sie im Norden finden. Ihr Auftreten beweist, $\mathrm{da} ß$ in einem bestimmten Zeitpunkte ein Ur-Bagmati das Valley querte und dieses Material bis hierher brachte. Nach oben folgen auch hier Mergel und weisen darauf hin, daß ein als Retentionsbecken wirkender See im Valley geschaffen wurde.

Damit gehören die Aufschlüsse im südlichsten Teil des Valley zu jenen, die besonders viel und zuverlässige Auskunft geben können. Die Bewegungen an der Scherfläche von Dakshinkhali haben auch die nördlich anschließenden Valleysedimente ver- 
Fig. 2 Geologisch-morphologische Übersicht

Fig. 3 Allgemeines Profil durch das Valley

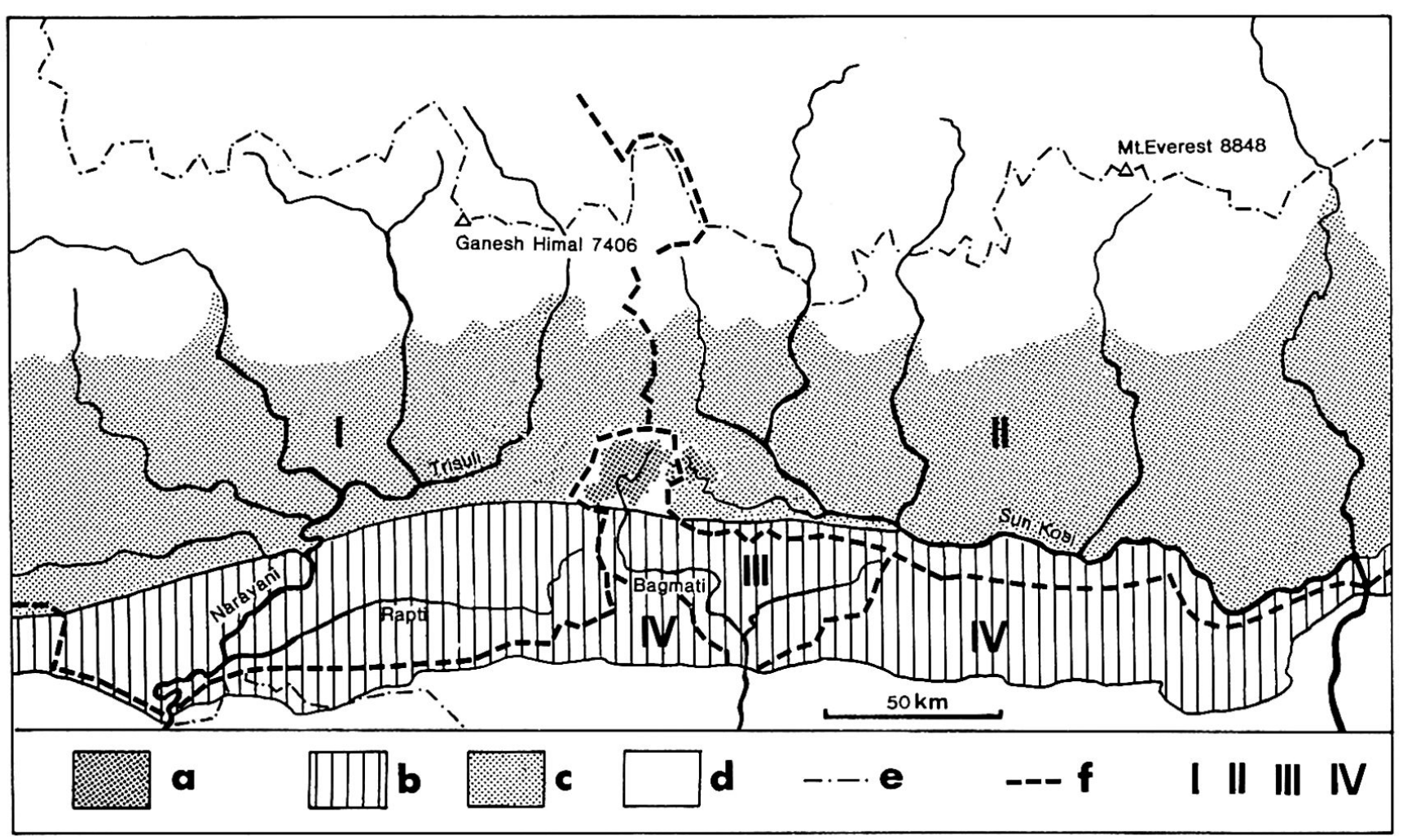

Fig. 2

Fig. 3

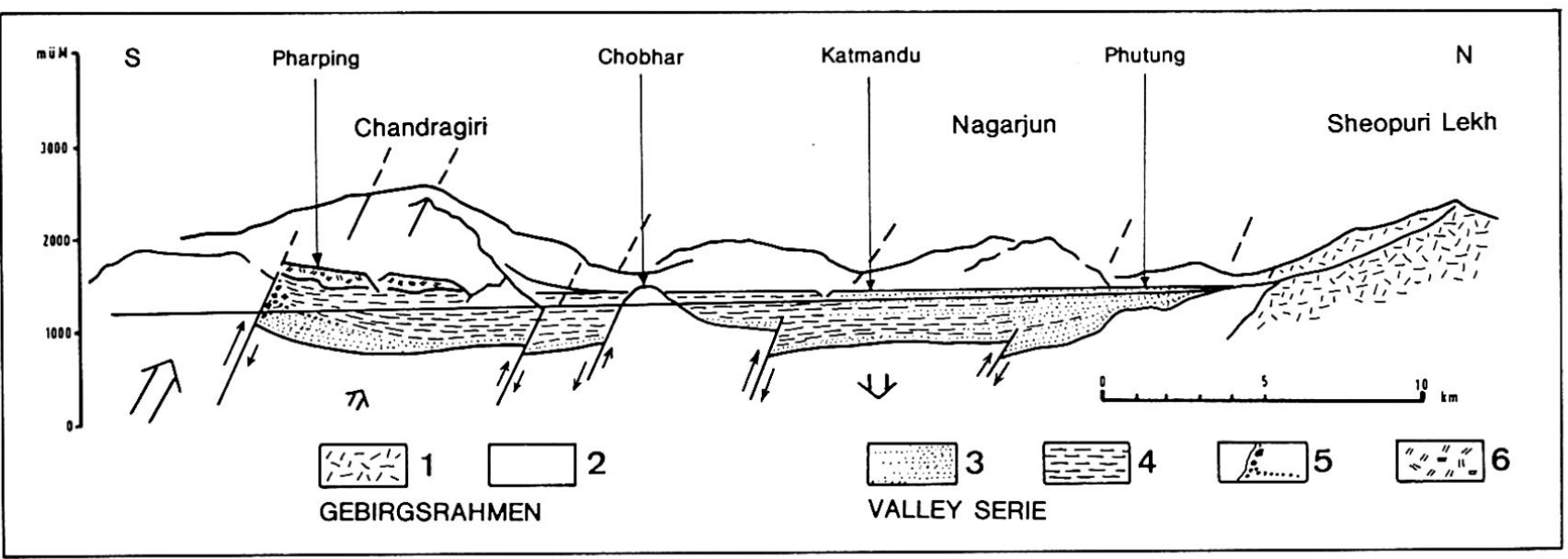


Fig. 4 Entwicklung des Entwässerungssystemes im Valley

Fig. 5 Schema der morphologischen Entwicklung

Fig. 4

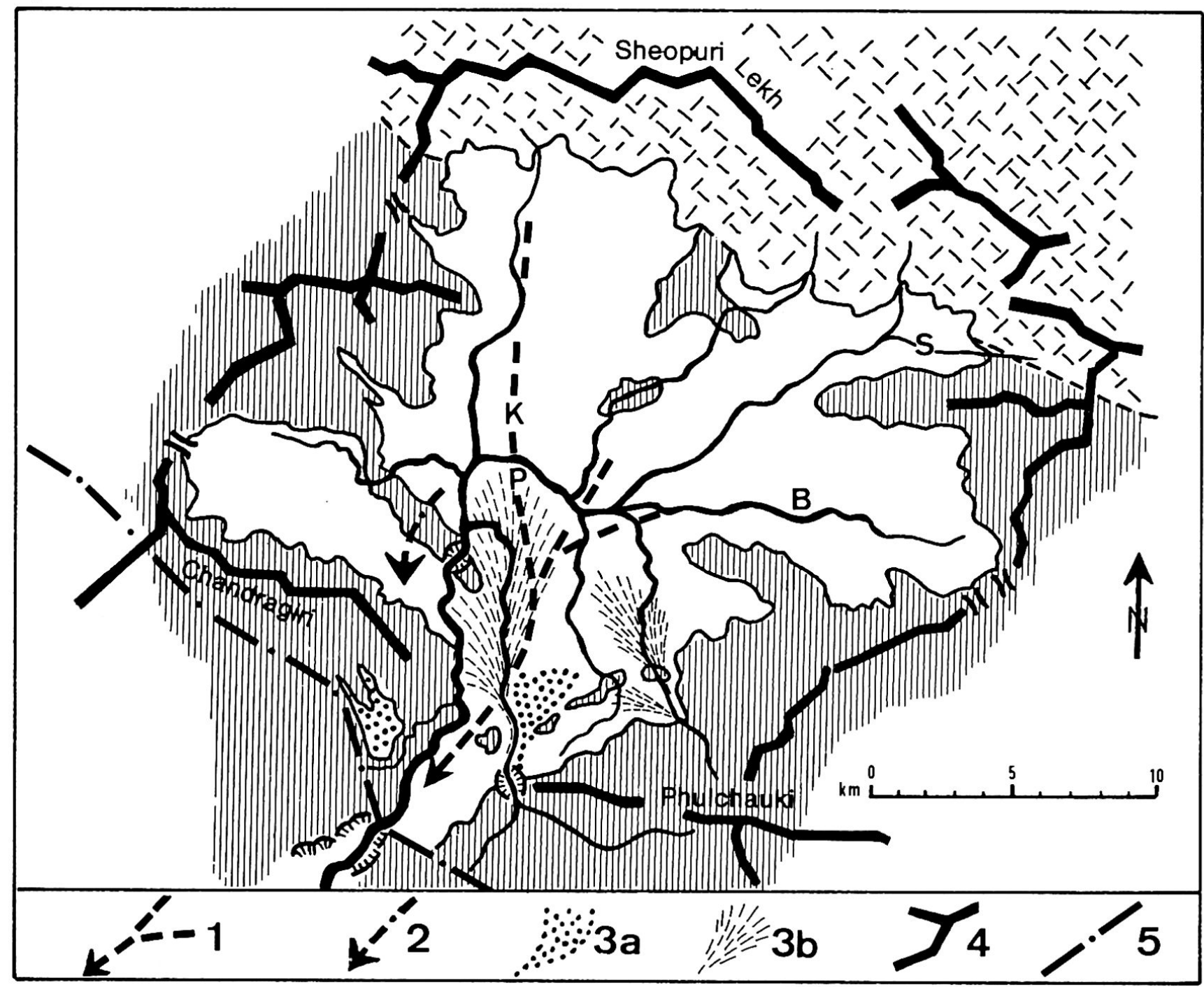

Fig. 5

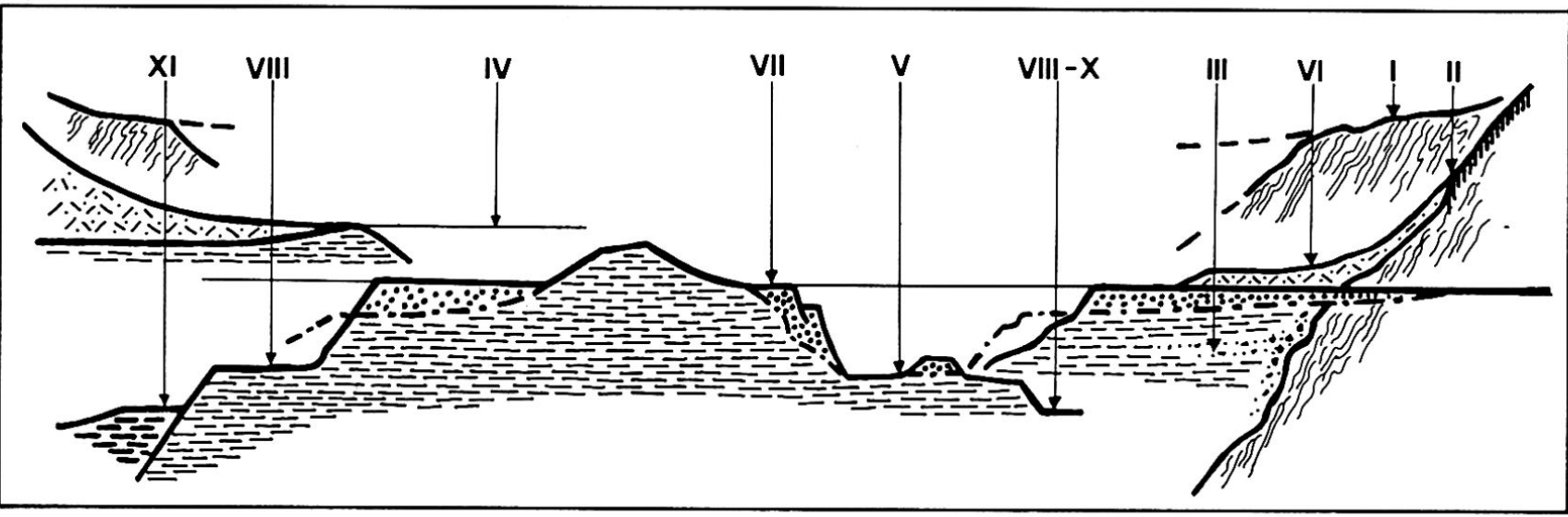


bogen. Mit $20-25^{\circ}$ fallen sie von der Scherfläche gegen Norden ein. Dabei kommen die Glimmersande bald unter den Talboden zu liegen. In den hangenden Mergeln läßt sich beobachten, daß diese Verbiegung nur lokal ist; schon wenige Kilometer von der Scherfläche entfernt liegen die Sedimente horizontal. Eine Bohrung war 1973 ungefähr 3 Kilometer von der Scherfläche flußaufwärts angesetzt worden in der Hoffnung, in geringer Tiefe die Glimmersande zu erreichen und dort einen artesischen Grundwasservorrat anzubohren. Zweifellos werden die Sande erreicht werden, dagegen schätzen wir die Möglichkeiten eines Grundwasserreservoirs aus noch zu nennenden Gründen sehr pessimistisch ein.

Wenn wir - in unserem Profil haben wir diese Auffassung zum Ausdruck gebracht - annehmen, daß hier die Phase der Glimmersande der Seebildung vorausging und ein durch kein Retentionsbecken unterbrochenes Entwässerungssystem im Valley dokumentiert, führten die folgenden Bewegungen an der Scherfläche zur Bildung eines tektonischen Stausees oberhalb dieses Punktes und zur Ablagerung von feinen Mergeln. In diesen wurden weiterhin von Norden Glimmersande bis höchstens nach Katmandu hineingeschwemmt, während im Süden der Godaveri Khola und Nakhu Khola Gerölle mehr oder weniger weit ins Valley hinaus zur Ablagerung brachten. Dazwischen finden sich weite Strecken ruhiger Küsten, wo die Mergel ungestört bis zum Anstehenden abgelagert werden. An der sich ständig bewegenden Scherfläche von Dakshinkhali dagegen wurden ununterbrochen oft viele Kubikmeter große Blöcke von Anstehendem in den ufernahen Partien abgelagert. Im untersten Teil, unmittelbar über den Glimmersanden, kam es sogar zur Ablagerung einer im Mittel 20 Meter mächtigen Konglomeratbank, die nordwärts unter den Talboden einfällt. Dies sind die Erscheinungsformen der sogenannten Randfazies (5) der Valleysedimente.

Die Seebildung wurde aber nicht allein durch die Bewegungen an der Scherfläche von Dakshinkhali kontrolliert. Die Ergebnisse der zahlreichen Bohrungen, ergänzt durch Interpretationen der Feldbeobachtungen, führen zur Annahme einer komplizierten Bruchtektonik im Valley. Die Felsbarriere an der Scherfläche liegt bei ca. $1185 \mathrm{~m}$ ü. M.; viele Bohrungen erreichen aber mit einer Ausgangshöhe von im Mittel etwa $1300 \mathrm{~m}$ ü. M. auch nach mehreren hundert Metern das Anstehende noch nicht. Die tiefsten bekannten Bohrungen sind jene von Harasidhi, welche ausgehend von $1326 \mathrm{~m}$ selbst in $427,20 \mathrm{~m}$ Tiefe das Anstehende noch nicht erreichten, und von Lagan Tole, die $476 \mathrm{~m}$ noch in den Valleysedimenten steckten. Der Feldbefund ergab ergänzend, daß beispielsweise die Felsrippe Kirtipur-Chobhar ein komplexes Gebilde ist, welches sich aus verschiedenen durch Brüche voneinander getrennten Elementen zusammensetzt. Die wichtigsten Bruchlinien verlaufen nach unserer Meinung - wie dies auch auf der geologischen Karte 1:63630 angegeben ist - mit angenähert nordwestlichem Streichen. Weil die Profilebene quer zu diesem Streichen verläuft, ist in der Zeichnung die Fortsetzung jeder Verwerfung im Hintergrund etwas nach rechts versetzt. Neben diesem Hauptbruchsystem ist ein zweites, quer dazu verlaufendes anzunehmen. Das ganze Valley erscheint damit als ein Bruchfeld. Die zum Teil viele hundert Meter mächtigen Mergelserien der Valleysedimente zeigen, daß diese durch Bruchlinien bestimmten Senkungsfelder zeitlich mit den stärksten Aufwärtsbewegungen an der Scherfläche zusammenfallen. Die Tatsache, daß die obersten Teile der Valleysedimente aber keine Verstellungen durch Brüche zeigen, deutet darauf hin, daß die tektonischen Bewegungen noch zur Zeit der Existenz des Katmandu-Sees mindestens im Valley selbst zum Abklingen kamen. Daß dies für den südlichen Teil nicht gilt, wird noch zu zeigen sein.

Mit dieser Bruchtektonik hängt auch die große Seismizität zusammen. Im Erdbeben von 1934 lagen die stärksten Zerstörungen auf einer Linie, die mit der großen Verwerfung in der Mitte unserer Figur zusammenfällt ${ }^{16)}$.

$\mathrm{Da}$ die Bewegungen sich auch nach erfolgter Auffüllung des Sees weiter fortsetzten, zeigt die Serie von Pharping (6). Es handelt sich bei dieser um meist sehr schlecht gerundeten Bachschutt, vermischt mit Roterden, der von zwei Bächen vom Chandragiri auf die Seesedimente hinausgeschwemmt und diesen aufgelagert wurde. Diese bis 30 Meter mächtige Schuttmasse bildet heute auf etwa 1500 m Höhe die Fläche von Pharping; durch spätere Erosion vom Bagmati her ist sie durch Bildung einer erosiven Steilstufe zu einer prägnanten Terrasse umgewandelt worden. Im Zusammenhang betrachtet 
läßt sich zeigen, daß auch diese späte Formation in zunehmendem Maße gegen Süden hin in die Höhe verbogen worden ist. Im Abschnitt von Pharping beträgt der Verbiegungsbetrag seit der Ablagerung der Serie (6) gut 150 Meter. Die früheren Hebungsbeträge an der Scherfläche sind mit 200-250 Metern anzusetzen, was im Total für die älteren Valleysedimente ein Total von 350-400 Metern ergibt. Diese starken jungen Verbiegungen sind auf den äußersten Süden beschränkt und lassen sich schon bei Chobhar nicht mehr nachweisen. Man muß freilich beachten, $\mathrm{da} ß$ Bewegungen in vertikaler Richtung mit diesen Methoden nie nachgewiesen werden können - man spricht dann in der Sprache der Geologen von einer ungestörten Lagerung.

Immer wieder wurde von uns auch die Frage gestellt, ob es tatsächlich zu einer Seebildung kam. Schon früher wurden aus den Mergelserien Beispiele von synsedimentären Rutschungen, wie sie nur subaquatisch vorkommen können, publiziert ${ }^{17)}$. Prächtige Deltaschüttungen bei Thimi und unweit Phutung beweisen ebenfalls stehende Wasserkörper. Die von uns früher eingehend behandelte Vivianitführung ${ }^{18)}$ konnte dagegen als Beweismittel nicht verwendet werden. Eine detaillierte paläontologischsedimentpetrographische Untersuchung der Valleysedimente wäre eine dankbare Aufgabe. An der Existenz eines stehenden Wasserkörpers ist nicht zu zweifeln. Sicher hat es sich dabei aber nicht um einen tiefen klaren Gebirgssee gehandelt, weit eher um ein in weiten Teilen amphibisches Sumpfgebiet. Darauf weisen auch die vielen Pflanzenreste führenden Lignitlager hin, die lokal sogar abgebaut werden.

Mit den früher erwähnten ersten Anlagen der Talsysteme, die über der heutigen Topographie zu denken sind, befassen wir uns hier nicht. Alle Talsysteme können erstmals in hochgelegenen Eckfluren und Gratlinien deutlich erkannt werden und sollten in regionalem Rahmen in Zukunft kartiert und interpretiert werden. In unserem Gebiet weist der $\mathrm{Zu}$ sammenhang dieser Formen auf ein mit dem heutigen kongruentes Flußsystem hin. Freilich steigen als Folge der erwähnten jungen Bewegungen diese alten Erosionssysteme südwärts an und sind auch an der Scherfläche verstellt. Wenn man aber die auf anderem Wege ermittelten Verbiegungsbeträge zur
Korrektur verwendet, erhält man ein dem heutigen Bagmati kongruentes südwärts gerichtetes Talsystem - dies obwohl die alten Erosionssysteme bedeutend höher als die Übergänge aus dem Valley, die wir früher erwähnten, liegen. Mit großer Klarheit kann man zwei ineinandergeschachtelte Erosionssysteme feststellen. Da dieser Teil des Himalaya nie vergletschert war, wird die Interpretation nicht wie in den Alpen durch glazialmorphologische Hypothesen erschwert.

Tiefer als diese frühen Systeme und tiefer als die Übergänge aus dem Valley nach Westen und Osten liegt eine charakteristische Oberfläche, welche wir das Roterde-Niveau nennen wollen. Dieses besteht aus den verschiedenartigsten Flächenelementen, Gehängen, Ebenen usw., die, wie nicht anders zu erwarten ist, in unterschiedlicher Höhe liegen. $\mathrm{Zu}$ sammen stellen sie eine alte Oberfläche dar, auf welcher praktisch ohne Rücksicht auf den Gesteinsuntergrund rote Böden gebildet wurden. Diese sind seit langem bekannt und oft beschrieben worden ${ }^{19}$. Meist sprach man von Lateriten. Das Roterde-Niveau ist sicher älter als die Valleysedimente; dies ergibt sich aus den Lagerungsverhältnissen. Roterdevorkommen im kartierten Gebiet sind auf Figur 1 eingezeichnet. Dabei handelt es sich im Gegensatz zur Serie von Pharping um Bildungen in situ. Verschwemmte Roterde, welche natürlich bedeutend jünger als das Roterde-Niveau sein kann, findet sich noch auf den 750-770 m ü. M. gelegenen Flußterrassen am Sun Kosi bei Dolalghat. Wir haben diesen Roterden besondere Aufmerksamkeit gewidmet und von zahlreichen Stellen Proben aufgesammelt, weil zu hoffen war, daß aus den Analysenergebnissen Rückschlüsse auf die paläoklimatischen Bedingungen zur Roterdezeit gezogen werden könnten. Möglicherweise repräsentieren sie eine spätpleistozäne (Riss/Würm) Warmzeit ${ }^{20}$ ).

Alle bisher genannten Entwicklungsstufen sind in der Figur 4 unberücksichtigt geblieben. Die Darstellung setzt mit der Entwässerung (1) ein, welche die Glimmersande quer durch das Valley an seinen südlichen Ausgang brachte. Die Chobhar-Schlucht wurde damals noch nicht benutzt, denn nirgends finden sich direkt südlich von Chobhar Glimmersande. 
(2) bezeichnet die Lage von zwei deutlich erkennbaren alten Überläufen über die Kirtipur-ChobharRippe. Sie erreichten nicht die Tiefe der heutigen Schlucht von Chobhar und wurden von Mergeln der Valleysedimente ausgefüllt. Diese Überläufe müssen damit nur während der Seebildung funktioniert haben und wurden später nicht mehr benützt. (3a) und (3b) sind Schotterfächer, welche als Resultat der im Süden andauernden Hebung und damit gesteigerten Erosionsintensität der Flüsse Nakhu und Godaveri ins Valley auf die Seesedimente hinaus vorgetragen wurden. Im Gegensatz zu früher fassen wir diese Intensivierung der Schotterführung nicht mehr als das Resultat einer Kaltzeit und damit klimamorphologisch ${ }^{21)}$, sondern als tektonisch bedingt auf. Die Kippungsfläche fällt gegen Nordosten und dies mag auch dafür verantwortlich sein, daß jeder Schuttfächer eine Tendenz zur Ausbreitung nach rechts zeigt und die Unterläufe des Godaveri Khola, Kodku Khola usw. alle nach rechts abbiegen. $\mathrm{Da}$ der Nakhu Khola während vieler Kilometer in genau entgegengesetzter Richtung zum Bagmati und von diesem nur 1 bis 2 Kilometer getrennt fließt, liegt darin, daß die epigenetische Chobhar-Schlucht als Erosionsbasis für alle Flüsse im Valley wirksam wurde. Erst unterhalb der Schlucht konnte sich der Bagmati rasch eintiefen (heutige Entwässerung 4), kontrolliert durch den Ausgang im Süden bei der Scherfläche von Dakshinkhali (5).

Eine zusammenfassende schematische Darstellung der gegenwärtigen Auffassung der Morphogenese des Valley gibt Figur 5. Unberücksichtigt blieben dabei jene frühen Phasen, welche nicht mehr in der gegenwärtigen Topographie erkennbar sind.

(I) sind Teile der eben besprochenen frühen Eintiefungssysteme wie Eckfluren und Verflachungen in mittleren und oberen Höhenlagen der den Rahmen des Valley bildenden Gebirge.

(II) ist eine in situ Roterde, welche durch ihre Lage die vermutlich Riss-Würm interglaziale Oberfläche festlegt. Roterden werden als Baumaterial (gestampfte Böden, Außenbemalung der Häuser) verwendet.

(III) sind die im See sukzessive abgelagerten Mergelserien. Die Randfazies mit Geröllen ist eingezeichnet. In diesem Profil fehlen dagegen die Glimmersande.
(IV) stellt das Ende (Akkumulationsniveau) der Seefüllung im Valley dar. Auf einem heutigen Niveau von $1375 \mathrm{~m}$ ü. M. laufen im Süden die Schwemmkegel vom Chandragiri, im Norden die jüngsten Glimmersandausschwemmungen von den Flanken des Sheopuri Lekh aus. In diesem Zeitpunkt war der See vermutlich fast völlig verlandet.

(IV-V) In diesem Zeitraum wurde der epigenetische Durchbruch bei Chobhar angelegt und Chobhar spielte erstmals seine Rolle als Erosionsbasis für die Valley-Entwässerung. Der See entleerte sich und die verschiedenen Flüsse schnitten sich in die Valleysedimente ein.

(V) bezeichnet das Ende dieser ersten Erosionsphase. Es läßt sich heute nicht mehr feststellen, ob diese mehrere Erosionsniveaus unfaßte und nur angenähert kann gesagt werden, daß die Erosion bis auf 20 Meter an das Niveau der gegenwärtigen Talausräumung heranreichte. Der Verlauf der Täler war zum mindesten im kartierten Gebiet mit dem heutigen identisch.

(VI) ist aus den Gebirgen ausgeschwemmter Gehängeschutt mit Geröllen, vermischt mit Roterden, welcher direkt den Valleysedimenten aufgelagert ist. Vermutlich handelt es sich bei der Auflagerungsfläche nicht um das Akkumulationsniveau (IV), sondern um ein kurz nachher folgendes Erosionsniveau. Eine eingehendere Untersuchung der Serie von Pharping könnte diesbezüglich eine Klärung bringen. Die in der Figur abgebildete Situation entspricht jener bei Chapagaon. Die Serie (VI) reichte nach den bisherigen Beobachtungen nie in dieTäler (V) hinein. Darin unterscheidet sie sich von den jüngeren Schottern (VII). Vor allem der Nakhu Khola mit seinem großen Einzugsgebiet und in geringerem Maße der Godaveri Khola brachten in dieser Phase große Mengen von Geröll in das Valley. Als Grund für die gesteigerte Geröllführung haben wir schon jungtektonische Bewegungen im südlichen Teile des Valley festgestellt. Diese Schotter füllten vorerst die in Phase (V) geschaffenen Täler auf und breiteten sich hierauf als weite Schotterfluren über die zwischen den Tälern liegenden Mergelflächen aus. Sie kommen vor allem bei Patan in großer Ausdehnung vor und wir nennen sie deshalb die Patan-Schotter ${ }^{22)}$. Das Akkumulationsniveau von (VII) liegt tiefer als (IV); aus diesem Grunde wird letzteres da und dort von flachen Mergelhügeln überragt. 
Diese Aufschotterungsphase deckte die Schlucht von Chobhar nicht ein; deren oberste Teile liegen höher als das Akkumulationsniveau von (VII). Sie diente deshalb auch während dieser Phase durchgehend als Erosionsbasis für das Valley und es kam nicht zu einer Änderung der Entwässerungsrichtungen (auf kleinere, wahrscheinlich tektonisch bedingte $\mathrm{Ab}$ lenkungen wurde schon hingewiesen). Nach Abschluß der Phase (VII) behielt darum die neue Erosionsphase die Talverläufe bei. Die Eintiefung erfolgte schrittweise (VIII, IX, X) und obwohl die Erosionsterrassen oft unscharf ausgebildet sind, kann man über dem heutigen Talniveau (X) sicher zwei Systeme unterscheiden. Da dies sowohl für den Abschnitt oberhalb wie unterhalb der ChobharSchlucht gilt, darf angenommen werden, daß das schrittweise Eintiefen tektonisch gesteuert wurde.

In den obersten Teilen von (IV) wurden von V. MITTRE $\mathrm{C}^{14}$-Bestimmungen vorgenommen ${ }^{23)}$, die mit einem Alter von rund 29000 Jahren zeigen, daß der Katmandu-See spät-würmeiszeitlich war. Nach in der Presse ${ }^{24)}$ erschienenen Mitteilungen hat derselbe Forscher aber auch bedeutend jüngere (ca. 5000 Jahre B.P.) Ablagerungen festgestellt, welche zusammen mit nachgewiesenen Getreidepollen für die neolithische Periode eine frühe Besiedelung des Valley belegen. In einer schriftlichen Mitteilung ${ }^{25)}$ hat Dr. V. MITTRE unsere Vermutung bestätigt, da $\beta$ diese Funde in den auf Figur 1 als Kalimati bezeichneten dunkeln Mergeln gemacht wurden und zwar in Straßeneinschnitten auf dem Wege von der Hauptstadt zur Universität. Während NAUTIYAL und SHARMA ${ }^{26)}$ noch annahmen, daß die Kalimati (XI) ein normales Schichtglied der Valleysedimente seien, müssen sie jetzt als sehr junge Einlagerungen in das bestehende Talsystem betrachtet werden. Sie sind jünger als die Patanschotter und füllen Erosionsformen der zweiten Eintiefungsphase aus. Ob tektonische Bewegungen oder allenfalls auch Abdämmung durch flußabwärts verstürzte Massen für die Aufstauung des Bagmati und die Sedimentation der Mergel verantwortlich waren, läßt sich zur Zeit nicht entscheiden. Diese wichtigen Altersbestimmungen legen aber nicht allein die Kalimati und die zweite Erosionsphase zeitlich fest, sondern gestatten auch, die Patanschotter und die Serie von Pharping in das Spät- und Postglazial zu stellen ${ }^{27)}$.

\section{Anmerkungen}

1) H. BOESCH: Morphologische Beobachtungen in Ost-Nepal, GH 1965; Das Katmandu Valley, Beiträge zur Morphologie von Nepal, GH 1968; Landnutzung und Geomorphologie im Katmandu Valley, Nepal, GH 1972; Die Brunnen von Patan (Lalitpur), Nepal, GH 1972. Es besteht keine einheitliche Schreibweise der Ortsnamen. Der Name der Hauptstadt wurde früher meist Kathmandu geschrieben, heute ist Katmandu üblich.

2) KESAR LALL: Lore and Legend of Nepal, Nagpur 1961.

Gopal Singh Nepali: The Newars-An Ethnosociological Study of a Himalayan Community, Bombay 1965.

3) P. C. Chakravatti: The Nepal Basin, Calcutta Geogr. Rev. 1945.

U. M. MALLA: Geomorphology of the Kathmandu Valley, The Himalayan Review 1971.

Auch der sehr substantielle Artikel von T. HAGEN: Über den geologischen Bau des Nepal-Himalaya mit besonderer Berücksichtigung der SiwalikZone und der Talbildung, Jb. St. Gall. Naturw. Ges. 1959, macht Angaben, die schwer überprüfbar sind.

4) Besonders wichtig waren

P.N. SHARMA und O. R. SINGH: Groundwater Resources of Katmandu Valley (Supplementary Report), Geol. Survey of India 1966.

S. P. NAUTIYAL und P. N. SHARMA: A Geological Report on the Groundwater Investigation of Kathmandu Valley, Geol. Survey of India 1961.

Ferner ein vorläufiger Bericht an WHO von der Firma Binnie and Partners, London, über: Water Supply and Sewerage for Greater Kathmandu and Bakhtapur, 1972.

5) HAGEN op. cit.

6) C. K. SHARMA: Geological Map of Nepal, $1: 1408000,1969$, HMG Katmandu. A. GANSSER: Geology of the Himalayas, Interscience Publ. 1964.

7) HAGEN op. cit. Fig. 2.

8) In der Zeitschrift «Mercedes-Benz, In aller Welt», XVIII 1973, p. 44-45, befindet sich eine sehr instruktive Flugaufnahme (Schrägaufnahme), 
welche die Verhältnisse im Bagmati-Tal unmittelbar oberhalb der Scherfläche von Dakshinkhali wiedergibt.

9) GANSSER op. cit., p. 153 (reproduziert nach J. B. AUDEN, 1935) HAGEN op. cit., Profil 30.

10) Nautiyal und sharma op. cit. Geol. Profil Gokarna-Balambu.

11) R. STAUB: Grundzüge und Probleme alpiner Morphologie, Denkschr. Schw. Naturf. Ges. LXIX/1 1934.

12) gegen Westen zum Manmati Khola 1524 m; nördlich von Nagarjun drei mit 1478, 1508 und 1479; gegen Banepa im Osten drei mit 1539, 1539 und 1551.

13) HAGEN op. cit. Fig. 10.

14) Geological Map of Kathmandu Valley $1: 63630$ by S. P. NAUTIYAL and P. N. SHARMA (vervielfältigt).

15) Einige Hinweise auf solche Berichte bei BoEsCH op. cit. 1968, p. 177.

16) J. B. AUDEN und A. M. N. GOSH: Preliminary Account of the Earthquake of the 15th January, 1934, in Bihar and Nepal, Geol. Survey of India (?) bes. p. 189, 191 und 202.

17) BOESCH op. cit. 1972, p. 177 Abb. 5.

18) BOESCH op. cit. 1968, p. 178-179.

19) Die Vorkommen bei Dolalghat beschreibt A. LOMBARD: Un itinéraire géologique dans l'Etat du Nepal (massif du Mount Everest), Denkschr. Schw. Naturf. Ges. Bd. 77/1, 1958. BOESCH op. cit. 1965 , p. 81, ergänzt und zitiert. Eine ausgezeichnete Flugaufnahme der sekundären Roterdevorkommen bei Dolalghat findet sich bei MARIO FANTIN: Sherpa Himalaya Nepal, Bologna 1971, Abb. 41.

20) Die Analyse der Proben wurde freundlicherweise vom Geographischen Institut der Universität Frankfurt/M übernommen. Die Interpretation der Analysenergebnisse ist schwierig und gesicherte Resultate liegen bis jetzt nicht vor. Es ist beabsichtigt, diese später in einer besonderen Arbeit über das Roterdesystem in GH zu publizieren.

21) BOESCH op. cit. 1968, p. 177.

22) BOESCH op. cit. 1972, p. 171-176.
23) Radiocarbon vol. 10/1, TF-189, Sankhu (Nepal): $29,115+3220 /-2285$. Peat from naturally exposed road-cutting on way to Sankhu $\left(27^{\circ} 43^{\prime}\right.$ N. Lat., $80^{\circ} 25^{\prime}$ E. Long.) near Kathmandu, Stratum upper peat-bed. Visible rootlets were handpicked. Subm. by A. GOSH. In der gegenwärtig im Druck befindlichen «Geology of Nepal» erwähnt C. K. SHARMA den Fossilfund bei Lukundol (Kokana) von Stegodon Ganesa, der ebenfalls pleistozänes Alter belegt. (Mündliche und schriftliche Mitteilung.)

24) Nepal Digest Oct./Nov. 1972, p. 5-6. Die dort erwähnten Untersuchungen wurden vom Birbal Sahani Institute of Paleobotany, Lucknow (Dr. V. MITTRE) ausgeführt.

25) Mitt. vom 20.8.1973; diese Angaben sind noch nicht publiziert.

26) NAUTrYAL und SHARMA op. cit., p. 9-10: The carbonaceous clay which is locally known as «Kalimati» has the largest spread towards the central part of the valley. It is overlain by sand and sandy clays in the central part of the valley, but much of the former has been removed by erosion, exposing the carbonaceous clays.

27) Nach Abschluß des Manuskriptes trafen die folgenden Publikationen ein, welche jedoch schon aufgrund mündlicher Mitteilungen teilweise berücksichtigt werden konnten:

C. K. SHARMA: Geology of Nepal, 189 S., geol. K., Kathmandu, 1973.

World Health Organisation-Project Nepal 0025: Appendix 4.4 Geology of the Kathmandu Valley und Appendix 4.5 Groundwater Investigations, Binnie and Partners, London, 1973 (ein umfangreicher Bericht mit vielen Karten und Tabellen).

Die Reinzeichnung der Figuren 1-5 wurde von Herrn Daniel Nüesch, dipl. Assistent am Geographischen Institut der Universität Zürich ausgeführt. Es ist dem Verfasser auch ein Bedürfnis, den Angehörigen der Schweizerischen Entwicklungshilfe SATA und den Herren Dr. Vishnu Mittre und Dr.C.K. Sharma für ihre wertvollen Mitteilungen, sowie Herrn Dr. H. Gurung für seine tatkräftige Unterstützung bei Regierungsstellen zu danken. 